\title{
Improved MIMO Signal Detection Based on DNN in MIMO-OFDM System
}

\author{
Jae-Hyun Ro ${ }^{1}$, Jong-Gyu Ha², Woon-Sang Lee ${ }^{2}$, Young-Hwan You ${ }^{3}$ and Hyoung-Kyu Song ${ }^{2, *}$
}

\author{
${ }^{1}$ Department of Convergence Engineering for Intelligent Drone, Sejong University, Seoul, 05006, Korea \\ ${ }^{2}$ Department of Information and Communication Engineering, Convergence Engineering for Intelligent Drone, Sejong \\ University, Seoul, 05006, Korea \\ ${ }^{3}$ Department of Computer Engineering, Convergence Engineering for Intelligent Drone, Sejong University, Seoul, 05006, \\ Korea \\ *Corresponding Author: Hyoung-Kyu Song. Email: songhk@sejong.ac.kr \\ Received: 30 May 2021; Accepted: 12 July 2021
}

\begin{abstract}
This paper proposes the multiple-input multiple-output (MIMO) detection scheme by using the deep neural network (DNN) based ensemble machine learning for higher error performance in wireless communication systems. For the MIMO detection based on the ensemble machine learning, all learning models for the DNN are generated in offline and the detection is performed in online by using already learned models. In the offline learning, the received signals and channel coefficients are set to input data, and the labels which correspond to transmit symbols are set to output data. In the online learning, the perfectly learned models are used for signal detection where the models have fixed bias and weights. For performance improvement, the proposed scheme uses the majority vote and the maximum probability as the methods of the model combinations for obtaining diversity gains at the MIMO receiver. The simulation results show that the proposed scheme has improved symbol error rate (SER) performance without additional receive antennas.
\end{abstract}

Keywords: MIMO; DNN; ensemble machine learning; ML

\section{Introduction}

In the future, studies aim to increase channel capacity in the overall wireless communication systems in order to handle explosive data traffic [1]. Multiple-input multiple-output (MIMO) technology has been studied in the wireless communication systems since tremendous increase of channel capacity is implemented [2-7]. In the MIMO systems, accurate signal detection is one of the main issues and various researches for the MIMO detection have been proposed for efficient signal detection [8-11]. Among various MIMO detection techniques, a maximum likelihood (ML) detection has optimal error performance. The ML detection has high complexity in huge MIMO system which has several antennas at the transmitter and uses high order modulation. When the constellation size is $L$ and the number of transmit antennas is $N_{t}$, the required number of metrics for calculating Euclidean distances is $L^{N_{t}}$. Thus, in the past, the main target of most researches in the MIMO signal detection was low-complexity algorithm which is practical in realtime wireless

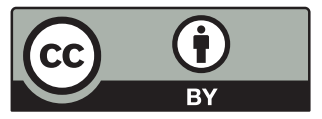

This work is licensed under a Creative Commons Attribution 4.0 International License, which permits unrestricted use, distribution, and reproduction in any medium, provided the original work is properly cited. 
communication systems. The already proposed algorithms had nearly optimal error performance where the gap of error performances compared with the ML detection is very small. Thus, this paper proposes new signal detector which has high error performance since an algorithm of the low-complexity detection is no more attractive. For performance improvement, this paper proposes a machine learning algorithm in the MIMO system.

The machine learning algorithm is very popular area and several technologies which use the machine learning algorithm in the MIMO systems have been studied in various fields of physical layer such as signal detection, channel estimation, and solution for non-convex problems [12-15]. Among several machine learning algorithms, the deep neural network (DNN) has a structure for dense-layer based several neurons. The DNN based MIMO signal detection is attractive since it does not require new algorithms regardless of transmitter structure and it can operate without complex mechanism when the number of learning data is enough. Also, the learning is performed in the offline in advance and the complete learning model can be used in the online. Surely, the learning model is not fitted in time-variant channel since the weights and biases which are calculated in the offline learning are not no longer valid, and it is impractical for updating these parameters according to quickly varied wireless channels. Nonetheless, this paper proposes the DNN based signal detection in time-invariant channels to show the possibility of MIMO signal detector based on the DNN which has high error performance despite of high complexity for the learning. These new trials are attractive in terms of high error performance since most researches were low-complexity signal detection which has nearly the same error performance as ML detection. However, the DNN based signal detection which has only one learning model at the receiver cannot have better error performance than the ML detection in a linear system. Thus, this paper proposes the DNN based ensemble machine learning for the MIMO signal detection which is a combination of several learning models and it can improve error performance when the learning models are accurately combined since the diversity gain is obtained. The proposed scheme introduces the encoding method of appropriate training data sets for the DNN generation and the structures for the MIMO signal detector based on the ensemble machine learning.

\section{MIMO Detection Technique}

\subsection{System Model}

This paper considers downlink single-user MIMO system where one base station which has $N_{t}$ transmit antennas communicates with one user which has $N_{r}$ receive antennas as shown in Fig. 1. The spatially multiplexed data streams are transmitted through the wireless channel and data streams are separated through the MIMO detection technique at the receiver. In this system model, the received symbols vector $\mathbf{y}$ is as follows,

$\mathbf{y}=\mathbf{H x}+\mathbf{n}$,

where $\mathbf{H}$ is a $N_{r} \times N_{t}$ complex Rayleigh flat fading matrix, $\mathbf{x}$ is a $N_{t} \times 1$ complex transmit symbols vector, and $\mathbf{n}$ is a $N_{r} \times 1$ complex additive white Gaussian noise (AWGN) vector. The statistical properties of all elements in $\mathbf{H}$ and $\mathbf{n}$ are zero-mean and unit variance, and the average power for $\mathbf{x}$ is normalized to 1 . 


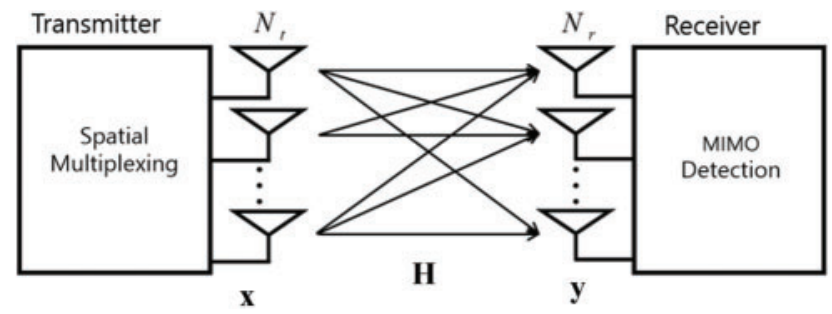

Figure 1: The $N_{r} \times N_{t}$ MIMO system model

\subsection{Conventional MIMO Detection Scheme}

This paper deals with the ML detection as a conventional scheme. Several low-complexity detectors which have optimal error performance have been studied. However, the past detectors are not considered as conventional schemes. The ML detection is performed by comparing the squared Euclidean distance between the received symbols and the combination of all reference symbols as follows,

$\mathbf{x}_{\mathrm{ML}}=\underset{\mathbf{x} \in \mathbf{X}}{\operatorname{argmin}}\left\{\|\mathbf{y}-\mathbf{H} \mathbf{x}\|^{2}\right\}$,

where $\mathbf{X}$ is set for all reference symbols. In several studies, the ML detection is a reference scheme for performance comparison with low-complexity algorithms since it has optimal error performance. Again, the low-complexity algorithms in the MIMO signal detection are no more attractive since a large number of low-complexity algorithms were proposed and performances are saturated to certain point. Thus, this paper proposes the DNN based ensemble machine learning algorithm in next section and these new trials show unprecedented performance improvement.

\section{Proposed MIMO DNN Based Ensemble Machine Learning}

This paper uses the ensemble machine learning algorithm in the MIMO detector for obtaining diversity gain. The error performance for the proposed scheme is improved compared with the detector which uses only one DNN model.

\subsection{Quick Overview of DNN}

The DNN can be viewed as a mapping function between the input and output. Therefore, the DNN describes a function as follows,

$\mathbf{o}=N N(\mathbf{i} ; \theta)$,

where the mapping function $N N(\cdot)$ is to find the best $\theta$ that maps $\mathbf{i}$ to $\mathbf{o}$. The weight parameter $\theta$ controls mapping function. Therefore, the DNN should find the optimal parameter $\theta^{*}$ that minimizes the difference between the output of DNN o and the given information $\mathbf{i}$. The weight $\theta$ is determined to minimize the loss function as follows,

$\theta^{*}=\underset{\theta}{\operatorname{argmin}} L(\mathbf{o}, \hat{\mathbf{o}})$,

where $L(\cdot)$ is a loss function.

The basic structure of the fully connected DNN is shown in Fig. 2. The fully connected DNN is composed of an input layer, several hidden layers and an output layer. 


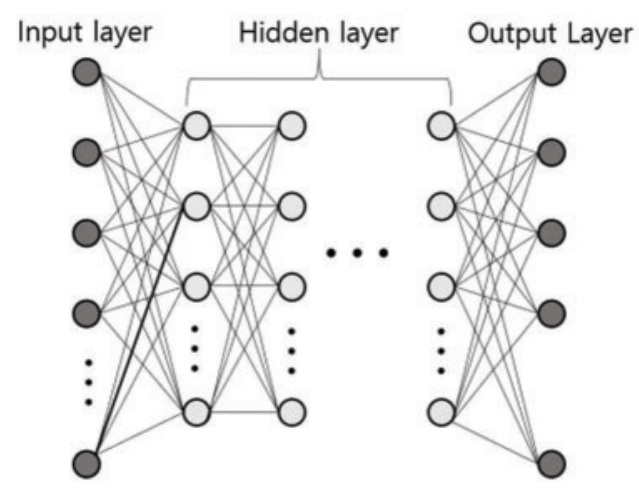

Figure 2: The basic structure of the fully connected DNN

\subsection{MIMO Detection Based on DNN}

The estimation of the transmit symbols from the received symbols is performed by using the DNN through supervised learning. The signal detection using the DNN in the MIMO system can be interpreted as a multi-class classification for detecting damaged symbols at the receiver. The accurate weights and biases in the DNN are generated through the offline learning which solves the problem between input and output values. For the MIMO detection using the DNN, $\mathbf{y}$ and $\mathbf{H}$ are input data, and a label for $\mathbf{x}$ is output data which is already known in the offline learning. In the DNN based MIMO system, the training data sets are generally complex number, and it has to be separated into real and imaginary numbers since all parameters in the DNN are real values. Tab. 1 represents an example of training data sets of the DNN when the number of total training data sets is $N$ where $\operatorname{Re}[\cdot]$ and $\operatorname{Im}[\cdot]$ represent the real part and the imaginary part of input data, respectively, and $h_{i j}$ is the channel coefficient from the $j$-th transmit antenna to the $i$-th receive antenna. The transmit symbols are encoded according to the number of transmit antennas and the used modulation order.

Table 1: The training data sets of proposed scheme

\begin{tabular}{llll}
\hline Data 1 & Data 2 & $\cdots$ & Data $N$ \\
\hline $\operatorname{Re}\left[y_{1}\right]_{1}$ & $\operatorname{Re}\left[y_{1}\right]_{2}$ & $\ldots$ & $\operatorname{Re}\left[y_{1}\right]_{N}$ \\
$\operatorname{Im}\left[y_{1}\right]_{1}$ & $\operatorname{Im}\left[y_{1}\right]_{2}$ & $\ldots$ & $\operatorname{Im}\left[y_{1}\right]_{N}$ \\
$\vdots$ & $\vdots$ & $\ddots$ & $\vdots$ \\
$\operatorname{Re}\left[y_{N_{r}}\right]_{1}$ & $\operatorname{Re}\left[y_{N_{r}}\right]_{2}$ & $\ldots$ & $\operatorname{Re}\left[y_{N_{r}}\right]_{N}$ \\
$\operatorname{Im}\left[y_{N_{r}}\right]_{1}$ & $\operatorname{Im}\left[y_{N_{r}}\right]_{2}$ & $\ldots$ & $\operatorname{Im}\left[y_{N_{r}}\right]_{N}$ \\
$\operatorname{Re}\left[h_{11}\right]_{1}$ & $\operatorname{Re}\left[h_{11}\right]_{2}$ & $\ldots$ & $\operatorname{Re}\left[h_{11}\right]_{N}$ \\
$\operatorname{Im}\left[h_{11}\right]_{1}$ & $\operatorname{Im}\left[h_{11}\right]_{2}$ & $\ldots$ & $\operatorname{Im}\left[h_{11}\right]_{N}$ \\
$\vdots$ & $\vdots$ & $\ddots$ & $\vdots$ \\
$\operatorname{Re}\left[h_{N_{r} N_{t}}\right]_{1}$ & $\operatorname{Re}\left[h_{N_{r} N_{t}}\right]_{2}$ & $\ldots$ & $\operatorname{Re}\left[h_{N_{r} N_{t}}\right]_{N}$ \\
$\operatorname{Im}\left[h_{N_{r} N_{t}}\right]_{1}$ & $\operatorname{Im}\left[h_{N_{r} N_{t}}\right]_{2}$ & $\ldots$ & $\operatorname{Im}\left[h_{N_{r} N_{t}}\right]_{N}$ \\
$\operatorname{Label~1}$ & $\operatorname{Label~} 2$ & $\cdots$ & $\operatorname{Label~} N$ \\
\hline
\end{tabular}


Tab. 2 represents an example of encoding of the transmit symbol in the $2 \times 2$ MIMO system when the quadrature phase shift keying (QPSK) modulation is used. The labels are set from 0 to 15 since the number of total reference symbols is 16 , and all labels are one-hot encoded for classification of transmit symbols. The training data set enters the input for the DNN learning, and goes through the rectified linear unit (ReLU) activation function at the hidden layer and the softmax function for multiple classification at the output layer. The ReLU function is the most commonly used function which greatly solves the problem of gradient descent problem. Finally, the number of neurons at the output layer corresponds to the number of labels. Each neuron at the output layer derives the probability that the label corresponding to the input signals through the softmax function is optimal. The output value of the softmax function is calculated by the cross-entropy cost function, and the weights and biases in the DNN are adjusted through a backpropagation algorithm based on the derived loss value. The overall learning process of the proposed scheme is shown in Fig. 3.

Table 2: An example of encoding of the transmit symbol in the $2 \times 2$ MIMO system with QPSK modulation

\begin{tabular}{lll}
\hline Data bit $(\mathrm{T} \times 1)$ & Data bit $(\mathrm{T} \times 2)$ & Label \\
\hline 00 & 00 & 0 \\
00 & 01 & 1 \\
00 & 10 & 2 \\
00 & 11 & 3 \\
01 & 00 & 4 \\
01 & 01 & 5 \\
$\vdots$ & $\vdots$ & $\vdots$ \\
11 & 11 & 15 \\
\hline
\end{tabular}

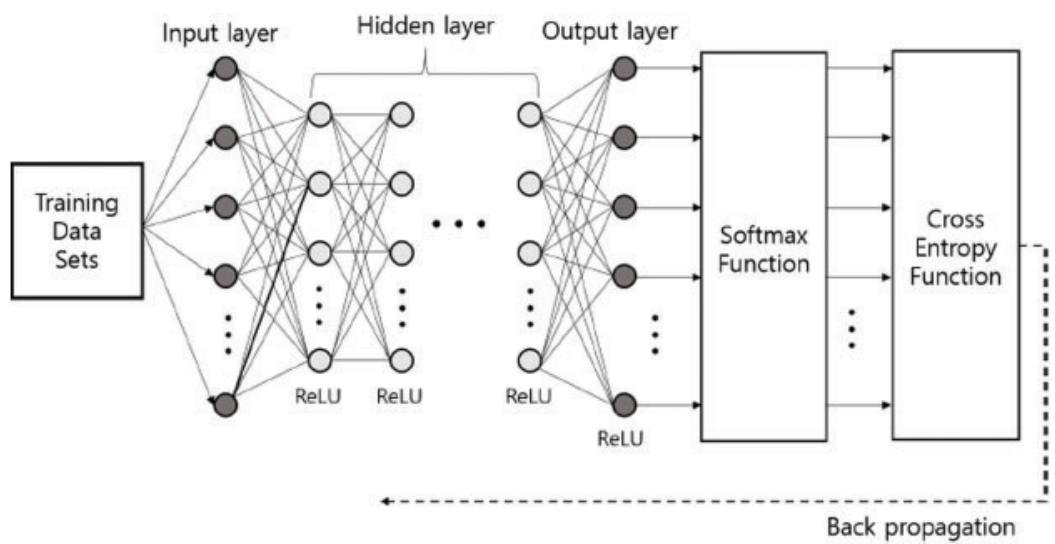

Figure 3: The overall learning process of the proposed scheme 
After the learning period, the DNN finally acts as one MIMO detector. The receiver inputs the received symbol and the channel states into the DNN based signal detection model to estimate the transmit symbols.

\subsection{MIMO Detection Based on Ensemble Machine Learning}

The proposed scheme uses multiple DNN models for obtaining additional performance gains. When the ensemble machine learning is used, the error performance for the MIMO detection is improved since the receiver obtains diversity gain by combining the results which are predicted by several different models. The ensemble method is an approach to make more informed decision which is made by combining multiple results from different models in an appropriate way. To implement the ensemble method, the two problems which generate multiple predictors and ensemble combination have to be considered. In the ensemble, there are many ways to construct different predictors. The property of individual classifiers which participate in ensemble combinations should be different to increase diversity gain. The proposed scheme uses random sampling and several DNN models with different structures. The random sampling repeats the random selection of training data to create several different training data sets. These training data sets create several classifiers. Therefore, different DNN structures lead $K$ networks to be independent with each other. In the prediction phase, the final result is determined mainly by using the method for majority vote or maximum probability. The majority vote selects the label with the most votes and the maximum probability selects the label with the highest value. For the majority vote, the output value of the $k$-th classifier when the number of classifiers is $K$ is as follows,

$\mathbf{o}_{V}^{(k)}=\left[\begin{array}{cccc}o_{V, 1}^{(k)} & o_{V, 1}^{(k)} & \cdots & o_{V, c}^{(k)}\end{array}\right]^{T}$,

where $o_{V, i}^{(k)}$ is the $i$-th one-hot encoded label and $c$ is the number of total labels.

For the output value, the majority vote selects the label with the most vote as follows,

$\hat{i}=\underset{i}{\operatorname{argmax}} \sum_{k=1}^{K} o_{V, i}^{(k)}$.

For the maximum probability, the probability of the label contains information for the models, and it can be used itself as information. The output value of the $k$-th classifier for the maximum probability is as follows,

$\mathbf{o}_{M}^{(k)}=\left[\begin{array}{llll}o_{M, 1}^{(k)} & o_{M, 1}^{(k)} & \cdots & o_{M, c}^{(k)}\end{array}\right]^{T}$,

where $o_{M, i}^{(k)}$ is the $i$-th probability of the estimation for each model.

The rule of the combination using the class probability is as follows,

$\beta_{i}=\max _{k} \mathbf{o}_{M}^{(k)}$. 
The proposed scheme calculates $\beta=\left[\begin{array}{llll}\beta_{1} & \beta_{2} & \cdots & \beta_{c}\end{array}\right]^{T}$ and selects the label with the highest value in $\beta$ as follows,

$\hat{i}=\underset{i}{\operatorname{argmax}} \beta_{i}$.

Fig. 4 shows a diagram which illustrates a combination of the DNN based MIMO signal detection. The $K$ learning models for the MIMO signal detectors are combined with each other to predict transmit symbols. The ensemble machine learning gives better performance compared to a single DNN model. The motivation of the proposed scheme is to exploit independence of each model since the basic approach for the signal detection is parallel combination of several learning models. As a result, the proposed scheme uses a single base algorithm to produce homogeneous detectors and it improves the error performance dramatically without additional receive antennas.

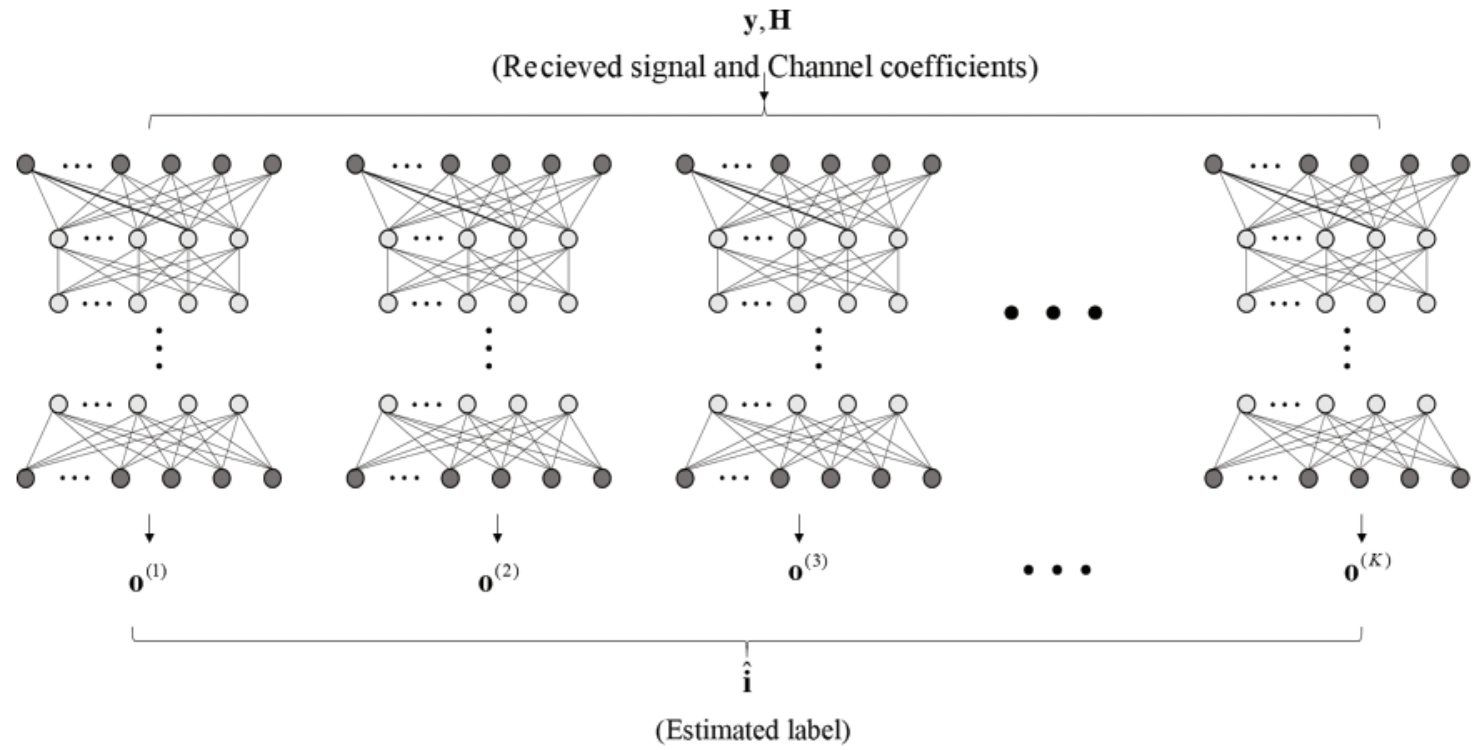

Figure 4: The ensemble machine learning based MIMO detection

\section{Simulation Results}

For evaluating the performance of the proposed scheme, symbol error rate (SER) and obtained diversity gain is measured. For performance evaluations, the training data which is a form of complex number is generated by MATLAB software and all models are learned by Keras library. The used channel model in the simulations is 7 multi-path Rayleigh fading. Finally, the simulations are performed on $2 \times 2$ and $3 \times 3$ MIMO systems which have small size compared with the number of maximally used antennas in long-term evolution (LTE) or wireless fidelity (Wi-Fi) systems, i.e., 8 antennas. However, the basic results for the MIMO signal detection using the model combination are not revealed clearly before. Also, the results of proposed algorithm in $2 \times 2$ and $3 \times 3$ MIMO systems can be enough for performance analysis and the proposed scheme can be extended to the large MIMO systems. 
Fig. 5 shows the SER performances for the conventional ML and the DNN based signal detection in $2 \times 2$ and $3 \times 3$ MIMO systems with QPSK modulation.

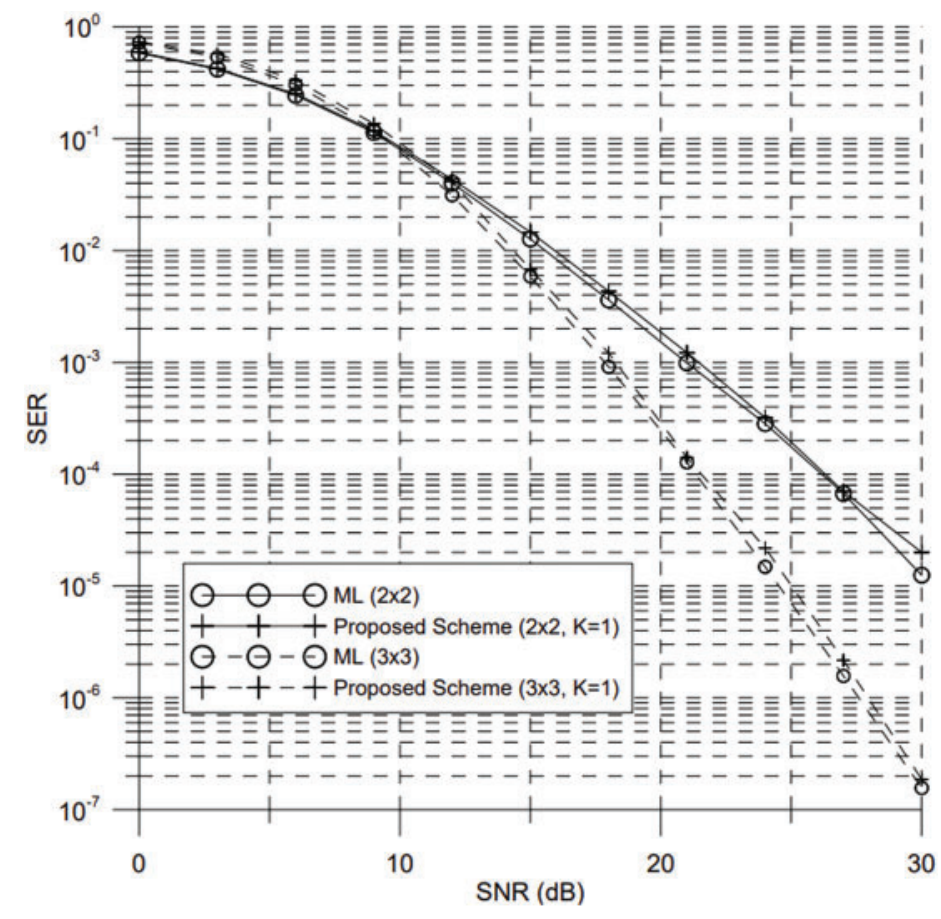

Figure 5: The SER performances for the conventional ML and DNN based detection in $2 \times 2$ and $3 \times 3$ MIMO systems with QPSK modulation

For performance comparisons, the SER performance for the conventional ML detection is shown. Again, this paper does not consider required complexity for showing improvement of the error performance clearly unlike existing algorithms. The DNN based signal detection is a special version of the proposed scheme with $K=1$ where the number of used models at the receiver is only one. Thus, the DNN based signal detection has similar SER performance with the ML detection since only one model is used and it gives no diversity gain. Also, the error performance for the DNN based signal detection is not superior to the ML detection in linear MIMO systems. In this section, the performance results in nonlinear fading are not shown since the main goal of this paper is to improve the error performance compared with the ML detection in linear MIMO systems.

Fig. 6 shows the SER performances for the proposed scheme with respect to the number of combined models in $3 \times 3$ MIMO system with QPSK modulation. The value of $K$ is set to 1,3 and 7 , respectively for clear visualizations of the performance improvement according to the increased number of $K$. Also, the simulation assumes a complete selection of the results from each model to show the performance improvement clearly. The SER performances for the proposed scheme with $K=3$ and $K=7$ are higher than the detector for the $K=1$ since the diversity gain is obtained at the receiver. The performance improvement is very attractive since the additional receive antennas are not used for the signal detection. Also, the SER performance 
for the proposed scheme is improved as the number of combined models increases since all generated models are independent each other.

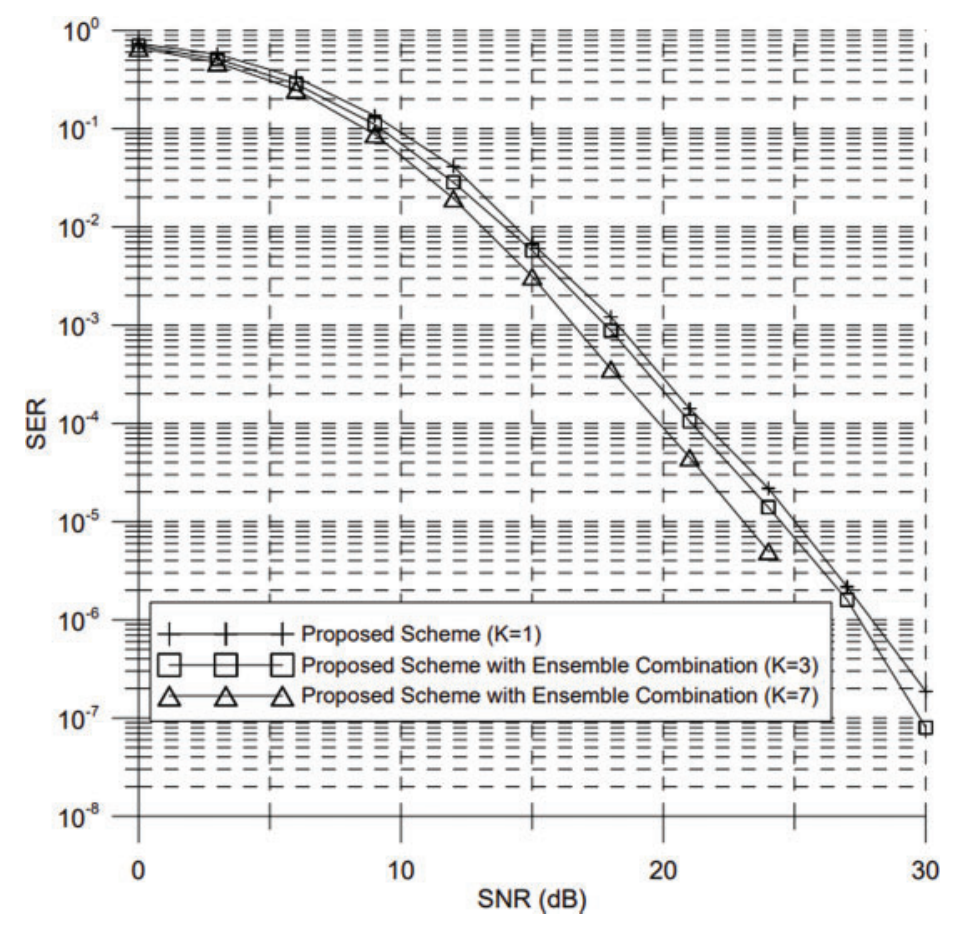

Figure 6: The SER performances for the proposed scheme with respect to the number of combined models in $3 \times 3$ MIMO system with QPSK modulation

Fig. 7 shows the SER performances for the proposed scheme $K=7$ according to different ensemble combination in $2 \times 2$ and $3 \times 3$ MIMO systems with QPSK modulation. In Fig. 7, the ensemble combinations for the majority vote and maximum probability methods are shown. The SER performances for the proposed scheme with model combination have better performance than the detector without model combination like results in Fig. 6. In $2 \times 2$ MIMO system, there is no large difference of the SER performance between the method for the majority vote and maximum probability. However, in $3 \times 3$ MIMO system, the method for the maximum probability has better SER performance than the method for the majority vote since the independence of the models is increased with each other due to the increased number of labels. These increases give more effect on the majority vote than the maximum probability.

For verification of performance results in Fig. 7, the obtained diversity gains are measured in Fig. 8 with same system parameters which are used in Fig. 7. In Fig. 8, the diversity gain is defined as the gain for the ensemble learning where it is calculated by dividing the value of each ensemble detector which shows different results. Therefore, the results in Fig. 8 show how much the gain is obtained by using the ensemble machine learning. The diversity gain is increased as the signal to noise ratio (SNR) increases, and the increased diversity gain leads to accurate signal detection in high SNR. 


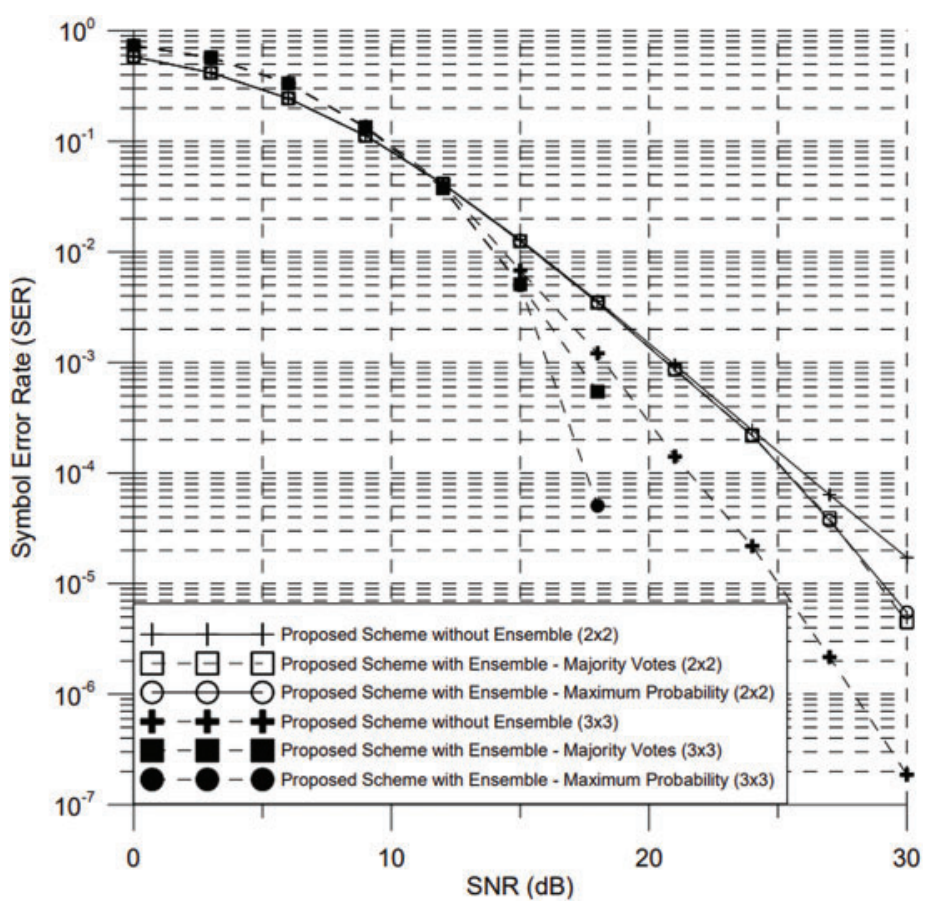

Figure 7: The SER performances for the proposed scheme according to different ensemble combination in $2 \times 2$ and $3 \times 3$ MIMO systems with QPSK modulation

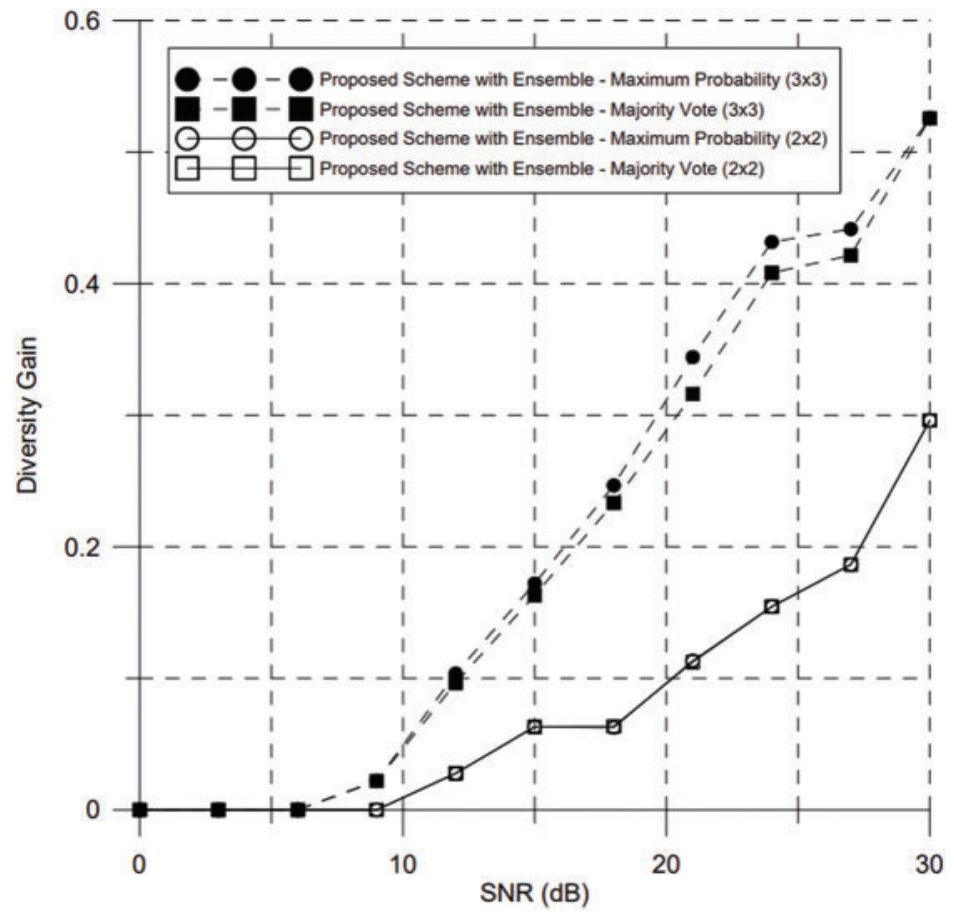

Figure 8: The measured diversity gain by ensemble combination method in $3 \times 3$ MIMO system

\section{Conclusion}

This paper proposes the ensemble machine learning based MIMO detection for high error performance. The proposed scheme suggests a possibility of machine learning in terms of perfor- 
mance gains in new aspects and solves the difficult part of conventional mathematical modeling and analysis in wireless communication. For the proposed scheme, the DNN based MIMO detector is introduced and the ensemble model is proposed for further improvement of the error performance. For efficient ensemble machine learning, the majority vote and maximum probability are used. The simulation results show that the proposed scheme has better SER performances than the conventional ML detection by obtaining diversity gain at the MIMO receiver. Also, the proposed scheme with the majority vote has better SER performance than the proposed scheme with the maximum probability. One of main advantages for the proposed scheme is performance improvement without additional receive antennas. Also, the proposed scheme can detect signals by using only data set. Thus, the proposed scheme can be effectively used as a highly reliable MIMO detector in wireless communication systems regardless of the structures of transmitter.

Funding Statement: This work was supported by the National Research Foundation of Korea (NRF) grant funded by the Korea government(MSIT) (No. NRF-2021R1A2C2005777) and was supported by Basic Science Research Program through the National Research Foundation of Korea (NRF) funded by the Ministry of Education (2020R1A6A1A03038540)

Conflicts of Interest: The authors declare that they have no conflicts of interest to report regarding the present study.

\section{References}

[1] A. Gupta and R. K. Jha, "A survey of 5G network: Architecture and emerging technologies," IEEE Access, vol. 3, pp. 1206-1232, 2015.

[2] R. Zi, J. Liu, L. Gu and X. Ge, "Enabling security and high energy efficiency in the internet of things with massive MIMO hybrid precoding," IEEE Internet of Things Journal, vol. 6, no. 5, pp. 8615-8625, 2019.

[3] A. Goldsmith, S. A. Jafar, N. Jindal and S. Vishwanath, "Capacity limits of MIMO channels," IEEE Journal on Selected Areas in Communications, vol. 21, no. 5, pp. 684-702, 2003.

[4] Z. Goldfeld and H. H. Permuter, "MIMO Gaussian broadcast channels with common, private, and confidential messages," IEEE Transactions on Information Theory, vol. 65, no. 4, pp. 2525-2544, 2019.

[5] G. Caire and S. Shamai, "On the achievable throughput of a multiantenna Gaussian broadcast channel," IEEE Transactions on Information Theory, vol. 49, no. 7, pp. 1691-1706, 2003.

[6] J. H. Ro, W. S. Lee, J. G. Ha and H. K. Song, "An efficient precoding method for improved downlink massive MIMO system," IEEE Access, vol. 7, pp. 112318-112326, 2019.

[7] J. G. Ha, J. H. Ro and H. K. Song, "Throughput enhancement in downlink MU-MIMO using multiple dimensions," Electronics, vol. 8, no. 7, pp. 758, 2019.

[8] J. H. Ro, S. J. Yu, Y. H. You, S. K. Hong and H. K. Song, "An adaptive QR-based energy efficient signal detection scheme in MIMO-OFDM systems," Computer Communications, vol. 149, no. 1, pp. 225-231, 2020.

[9] B. S. Kim, S. D. Kim, D. J. Na and K. H. Choi, "A very low complexity QRD-M MIMO detection based on adaptive search area," Electronics, vol. 9, no. 5, pp. 756, 2020.

[10] S. J. Choi, S. J. Shim, Y. H. You, J. S. Cha and H. K. Song, "Novel MIMO detection with improved complexity for near-ML detection in MIMO-OFDM systems," IEEE Access, vol. 7, pp. 60389-60398, 2019.

[11] S. J. Shim, S. J. Choi and H. K. Song, "Low power consumption signal detector based on adaptive DFSD in MIMO-OFDM systems," Energies, vol. 12, no. 4, pp. 599, 2019.

[12] T. O'Shea and J. Hoydis, "An introduction to deep learning for the physical layer," IEEE Transactions on Cognitive Communications and Networking, vol. 3, no. 4, pp. 563-575, 2017. 
[13] N. Samuel, T. Diskin and A. Wiesel, "Deep MIMO detection," in 2017 IEEE 18th Int. Workshop on Signal Processing Advances in Wireless Communications, Sapporo, Japan, pp. 1-5, 2017.

[14] Z. Qin, H. Ye, G. Y. Li and B. F. Juang, "Deep learning in physical layer communications," IEEE Wireless Communications, vol. 26, no. 2, pp. 93-99, 2019.

[15] H. He, C. Wen, S. Jin and G. Y. Li, "A model-driven deep learning network for MIMO detection," in 2018 IEEE Global Conf. on Signal and Information Processing, Anaheim, CA, USA, pp. 584-588, 2018. 